\title{
¿Son realmente tan buenos los nativos digitales? Relación entre las habilidades digitales y la lectura digital
}

\author{
Inmaculada Fajardo, Ester Villalta y Ladislao Salmerón*
}

Universitat de València (España).

\begin{abstract}
Resumen: La competencia en la lectura digital consiste en la comprensión, uso, reflexión y disfrute de los textos escritos con el fin de conseguir nuestros objetivos, desarrollar nuestro conocimiento y potencial, y participar en nuestra sociedad. En la actualidad se considera que los "nativos digitales", aquellos estudiantes que desde la infancia han crecido rodeados de las tecnologías de la información, poseen las habilidades digitales básicas (usar el ratón, el navegador, ...) necesarias para desarrollar la competencia en la lectura digital. En el presente estudio ponemos a prueba esta visión, a partir de un estudio en el que estudiantes de $5^{\circ}$ de Primaria y $3^{\circ}$ de Secundaria realizaron una serie de tareas de lectura digital. Los estudiantes completaron asimismo varias pruebas objetivas para medir sus habilidades digitales básicas, así como si nivel de competencia lectora en papel. Los resultados mostraron que los estudiantes no sólo presentaban dificultades en numerosas habilidades digitales básicas, si no que éstas estaban relacionadas directamente con el éxito en tareas de lectura digital. Para concluir, se reflexiona sobre la necesidad de considerar la instrucción en las habilidades digitales básicas como parte de los esfuerzos actuales por mejorar la competencia en la lectura digital.

Palabras clave: Habilidades digitales; lectura digital; comprensión lectora;
\end{abstract} educación primaria; educación secundaria.

\section{Introducción}

La realidad actual impone el uso de las TIC y entre ellas, el uso de Internet como medio de adquisición del conocimiento frente a metodologías educativas tradicionales basadas en la lectura en papel. De hecho, tal como apunta Ángel Gurría, Secretario General de la Organización para la Cooperación y el Desarrollo Económicos (OECD, 2011, pág. 5): “El mundo es indiferente hacia la tradición y las glorias del pasado, es implacable ante la fragilidad, la complacencia y la ignorancia de la práctica por costumbre. El éxito será para aquellos individuos y países que sean rápidos en adaptarse, lentos en quejarse y abiertos al cambio". Ante este reto educativo, es necesario considerar cómo lograr la competencia en la lectura digital, entendida, tal como se expone en el marco PISA 2009, como la comprensión, uso, reflexión y disfrute de los textos escritos con el fin de conseguir nuestros objetivos, desarrollar nuestro conocimiento y potencial y participar en nuestra sociedad. En España existe el propósito de introducir las Tecnologías de la Información y el Conocimiento (en adelante TIC) en el ámbito educativo con el objetivo de igualar los parámetros que definen una Sociedad de la comunicación al ámbito europeo. Siguiendo esta política, se han implementado programas públicos que han logrado que un $85 \%$ de los centros de enseñanza no universitaria soste-

* Dirección para correspondencia [Correspondence address]: Ladislao Salmerón. Departamento de Psicología Evolutiva y de la Educación. Universitat de València. Avd. Blasco Ibáñez, 21. CP 46010, Valencia (España). E-mail: Ladislao.Salmeron@uv.es

\begin{abstract}
Title: Are really digital natives so good? Relationship between digital skills and digital reading.

Abstract: Digital reading literacy consists on the comprehension, use, reflection and enjoyment of written texts with the aim to fulfill our goals, to develop our knowledge and potential, and to participate in our society. Currently it is considered that "digital natives", i.e. those students that have been raised surrounded by information technologies, poses the basic digital skills (such as using the mouse, the browser, ...) required to develop digital reading skills. The present study tested this assumption, by means of a study in which students of 5th level of primary education and 3rd level of secondary education performed a series of digital reading tasks. In addition, students completed several objective tasks to measure their basic reading skills, and their printed reading comprehension. The results revealed that the groups assessed had difficulties in several basic digital skills, and that those skills are directly related to success rate in digital reading tasks. A regression analysis revealed that this relationship was independent of students' printed reading skills, as well as of students' navigation during the digital reading tasks. To conclude, we reflected on the need to consider the instruction of basic reading skills as part of the current efforts to improve digital reading literacy.
\end{abstract}

Key words: Basic digital skills; digital reading; reading comprehension; primary education; secondary education.

nidos con fondos públicos tengan acceso a internet (Muriel, 2009).

Conseguido el reto inicial de introducir la tecnología en las aulas, una asignatura pendiente es determinar qué tipo de instrucción necesitan los estudiantes de educación primaria y secundaria para alcanzar los retos planteados por la lectura digital. Este trabajo pretende explorar hasta qué punto las habilidades digitales para interactuar con los medios electrónicos, como usar el ratón, los botones avance y retroceso de un navegador web, el scroll para acceder a la parte inferior de la pantalla o clicar hiperenlaces, son esenciales para el éxito en la lectura digital, y por tanto deben ser objeto importante de instrucción.

Este punto suscita grandes polémicas, y de hecho existe una concepción muy enraizada tanto en la comunidad científica como en el ámbito educativo que considera que los estudiantes de educación primaria y secundaria de hoy en día no necesitan ningún tipo de instrucción para interactuar con los medios electrónicos. El argumento esgrimido es que estos jóvenes pertenecen a una generación de nativos digitales que utilizan constantemente las tecnologías de la información, y que por tanto dominan ampliamente esta serie de habilidades digitales básicas (Prensky, 2001). Sin embargo, la visión de los 'nativos digitales' ha sido recientemente criticada por sobrevalorar las habilidades digitales de los estudiantes actuales, particularmente de aquellos de educación primaria y secundaria (Bennett, Maton y Kervin 2008; Bennett y Maton, 2010). Según estos autores, de la revisión de los estudios sobre el uso de la tecnología de los estudiantes actuales se desprende que la mayoría de jóvenes sólo realizan con 
frecuencia unas pocas actividades digitales, como son el acceso a información y la comunicación vía internet o dispositivos móviles. La realización de otras actividades propias de la Web 2.0, como la creación de contenidos o la publicación de información, varía enormemente dentro de esta población. No podemos hablar, por tanto, de una generación uniforme de estudiantes expertos en el manejo de los entornos digitales. Pero para determinar la importancia de enseñar estas habilidades queda por determinar, en cualquier caso, hasta qué punto la variabilidad en las habilidades digitales tiene un impacto significativo en las tareas de lectura digital.

\section{Habilidades digitales y lectura digital}

¿Qué papel juegan las habilidades digitales para el manejo de la tecnología en el éxito en la lectura digital? Para responder a esta pregunta, Van Deursen y Van Dijk (2009) proponen un modelo de cuatro competencias básicas necesarias para tener éxito la lectura digital: habilidades operativas y formales de internet y habilidades de información y estratégicas. Las dos primeras serían habilidades propias del medio, es decir, de Internet (lo que en este documento llamaremos "habilidades digitales"), como saber usar botones de back y forward de un navegador o conocer las estructura de un sitio Web. Las habilidades de información y estratégicas, se refieren a la comprensión de contenidos propiamente dicha y la puesta en práctica de estrategias de solución de problemas, como la evaluación de las fuentes de credibilidad o la adopción de rutas de navegación hipertextual (cf. Modelo de resolución de problemas en internet, de Brand-Gruwel, Wopereis y Walraven, 2009). Para testar su modelo, Van Deursen y Van Dijk (2009) diseñaron un estudio usando una muestra de usuarios adultos (de 18 a 74 años). Los participantes resolvieron una serie de tareas cotidianas usando Internet. Mientras que unas tareas medían habilidades digitales (e.g. acceder a una página web, guardar la dirección de una web en favoritos), otras eran tareas propias de la lectura digital tal y como las define PISA -ver definición más arriba(e.g. usando Google, encontrar el ticket de avión más barato para volar a Londres en unas fechas concretas). Se midieron los aciertos, el número de acciones realizadas para conseguir los objetivos y el tiempo dedicado. Los resultados mostraron que la ejecución en las tareas de habilidades digitales predecía positivamente la ejecución en las tareas de lectura digital independientemente de otras variables relevantes como las horas de uso de Internet, el nivel educativo, o la edad.

Aunque el estudio de Van Deursen y Van Dijk (2009) demuestra la importancia de las habilidades digitales en la lectura digital en adultos, debemos ser cautos a la hora de extrapolar estos resultados a los estudiantes no universitarios. De hecho, son pocos los estudios sobre lectura digital con estudiantes de educación primaria o secundaria que evalúan las habilidades digitales. Y en la mayoría de los casos, dichas habilidades se analizaron de forma indirecta a partir de indicadores como la frecuencia de uso del ordenador o el número de años de experiencia con los ordenadores (Coiro y
Dobler, 2007; OECD, 2011; Tu, Shih y Tsai, 2008). Pero el uso de estas medidas presenta varios problemas, ya que no son necesariamente fiables y pueden dar lugar a múltiples interpretaciones. Por ejemplo, en el estudio de lectura digital de PISA 2009 (OECD, 2011) se encontró que en varios países los jóvenes que usaban el ordenador en casa para tareas escolares muy poco (cuartil inferior) o mucho (cuartil superior) obtenían peores resultados que los que usaban el ordenador de forma intermedia (cuartiles intermedios). Si las habilidades digitales pueden inferirse a partir de la frecuencia de uso de ordenadores en casa, ¿cómo es posible un patrón de resultados como este? Los autores sugieren que los estudiantes que usan mucho el ordenador en casa para las actividades escolares puede que sean menos aventajados y por ese motivo necesiten más tiempo para realizar las tareas. En otras palabras, la frecuencia de uso de los ordenadores incluye más componentes que las habilidades digitales, por lo que de la literatura existente no se puede determinar de forma fiable la relación de dichas habilidades con la lectura digital.

Hasta donde hemos podido comprobar, los únicos estudios que han analizado las habilidades digitales de estudiantes de secundaria mediante una tarea objetiva, así como su impacto sobre tareas de lectura electrónica en el marco de PISA, son los recientes trabajos de Goldhammer, Naumann y Keßel (2013) y Hahnel, Goldhammer, Naumann y Kröhne (2016). Los autores encontraron que el test de habilidades digitales predecía un aproximadamente un tercio de la varianza de los aciertos a las preguntas de lectura digital en una muestra de estudiantes con una edad media 15 años (los cursos específicos analizados no se reportan). A partir de estos resultados los autores concluyen que las habilidades digitales básicas son un prerrequisito para realizar con éxito la lectura digital. Desgraciadamente para estos análisis los autores no controlaron dos de los factores que han demostrado ser esencial para explicar el éxito en las tareas de lectura digital en el marco de PISA: el nivel de comprensión lectora de los estudiantes, y la navegación durante la lectura digital (Coiro, 2011; OECD, 2011; Salmerón y García, 2011).

En cuanto al primero de los factores, investigaciones previas han constatado que los alumnos que son buenos identificando ideas textuales, estableciendo inferencias entre ideas relacionadas, o sintetizando información, son también buenos navegando y respondiendo a las tareas de lectura digital (Coiro, 2011; OECD, 2011; Salmerón y García, 2011). Por tanto, queda abierta la posibilidad de que el efecto de las habilidades digitales esté parcial o totalmente explicado por las habilidades de comprensión de los estudiantes. Por otro lado, los autores sólo evaluaron a estudiantes de 3 de ESO, por lo que sus resultados no son fácilmente generalizables a estudiantes de cursos inferiores, en los que los niveles tanto de comprensión lectora como de habilidades digitales puede variar enormemente.

En cuanto a la navegación, estudios previos con tareas similares a las propuestas por PISA han revelado que los estudiantes de educación secundaria que seleccionan páginas con información relevante para resolver la tarea a la vez que 
evitan páginas irrelevantes obtienen una mejor puntuación en las tareas de lectura digital (OECD, 2011). La falta de control de esta variable en el estudio de Goldhammer y cols (2013) impide conocer si el impacto de las habilidades digitales se debe a que facilitan la navegación en páginas relevantes, o si su efecto es independiente de ésta (e.g. el uso eficiente del scroll podría ayudar a integrar información dispersa dentro de una misma página).

El presente estudio sigue la línea de los estudios revisados para evaluar la relación entre habilidades digitales y lectura digital en estudiantes de educación primaria y secundaria. Con tal fin se evaluaron tanto las habilidades digitales como de lectura digital de un grupo de estudiantes de $5^{\circ}$ de Educación Primaria y $3^{\circ}$ de ESO. Como objetivos específicos, en primer lugar se pretenden replicar los efectos del nivel de comprensión lectora, habilidades digitales y navegación en las tareas de lectura digital. En segundo lugar, se pretende determinar si el efecto de las habilidades digitales en las tareas de lectura digital es independiente de los efectos de comprensión lectora y de la navegación, tal y como se desprende del modelo de Van Deursen y Van Dijk (2009).

\section{Método}

\section{Participantes}

En el estudio participaron 50 estudiantes, de dos aulas de $5^{\circ}$ de Primaria $\left(N=19,10-11\right.$ años, $47 \%$ niñas) y $3^{\circ}$ de ESO ( $N=31,14-15$ años, $48 \%$ niñas). El colegio, situado en la localidad de Paterna (Valencia), es de tipo concertado y forma parte de una red de colegios a nivel nacional. Las familias de los estudiantes del centro son generalmente de nivel socioeconómico medio. Los alumnos integrados o que reciben atención psicopedagógica participaron en el estudio pero sus resultados no han sido incluidos en la muestra de 50 alumnos. Se solicitó y obtuvo el consentimiento paterno por escrito a todos los alumnos participantes.

Se recabó información sobre la experiencia con los ordenadores y con Internet de los participantes a partir del cuestionario elaborado por Richter, Naumann y Horz (2010). Los estudiantes de ambos cursos tienen experiencia de varios años con el uso de ordenadores (Primaria: $M=3.1, D T=$ 1.7; ESO: $M=5.4, D T=1.9$ ), siendo el uso en casa -horas semanales- más frecuente en el caso de los estudiantes mayores (Primaria: $M=3.5, D T=2.4$; ESO: $M=10.8, D T=$ 8.6). La mayoría de estudiantes utiliza el ordenador en casa tanto para estudiar como para el ocio (Primaria: 80\%; ESO: $90 \%$ ). En cuanto a los programas más utilizados, los estudiantes de Primaria usan el ordenador sobre todo para juegos $(90 \%)$, seguido a gran distancia de la edición de textos $(30 \%)$. Por su parte, los estudiantes de ESO usan en su mayoría el ordenador para la edición de textos $(90 \%)$, seguido de la edición de imágenes $(70 \%)$, la elaboración de presentaciones $(70 \%)$, y en menor medida los juegos (50\%), edición de video $(50 \%)$ y de audio $(50 \%)$. Por último, en cuanto a los usos de Internet, los estudiantes de Primaria fundamen- talmente lo usan para buscar información ( $80 \%$ ) y jugar en línea $(60 \%)$, seguido de la descarga de música $(40 \%)$ y el visionado de vídeos (40\%). Los estudiantes de ESO, por su parte, en su mayoría usan Internet para el uso de redes sociales $(90 \%)$, descarga de música $(90 \%)$ y el visionado de vídeos $(90 \%)$, seguido de la búsqueda de información (80\%), los juegos en línea $(80 \%)$, y la lectura del correo electrónico $(70 \%)$.

\section{Materiales}

Portal web

Se construyó un portal Web para esta investigación con contenidos (textos) adaptados a estudiantes adolescentes que se extrajeron de otras páginas existentes. La estructura del sitio Web fue de tipo jerárquico con 4 niveles de páginas o nodos. Siguiendo los criterios de PISA (OECD, 2009) se diseñaron 9 hipertextos discontinuos (con tablas, imágenes, videos...) y 22 hipertextos continuos (únicamente con información textual). Se controló el número de palabras de los textos de manera que no sobrepasaran las 500 palabras. Por cada subsección, dos de los tres hipertextos iban acompañados de elementos distractores que aunque relacionados temáticamente no aportaban información adicional sobre el texto (videos o imágenes). Los enlaces podían tener dos formatos. Por un lado, el menú principal que se presentaba a la izquierda representaba el primer nivel de navegación, con las categorías de las distintas secciones (e.g., medioambiente, salud.....). Por otro lado, en las páginas de contenidos se presentaban un segundo y tercer nivel de navegación. En el segundo, los enlaces estaban formados por más de una palabra (e.g., material deportivo, animales protegidos...), mientras que en tercero aparecían los títulos de los hipertextos de mayor longitud (e.g., la elección de jugadores en la NBA). El sistema impedía avanzar más allá, por lo que los enlaces en los hipertextos del tercer nivel fueron deshabilitados.

\section{Tareas de lectura digital}

Se diseñaron tres tipos de preguntas de opción múltiple con tres alternativas de respuesta sobre el contenido del hipertexto, siguiendo el esquema propuesto por PISA (OECD, 2009): las preguntas de localización implican acceder y localizar información; las preguntas de integración requieren integrar e interpretar información y las preguntas de reflexión implican evaluar e ir más allá del texto (ver tabla 1 para un análisis detallado de las tareas). Seis preguntas consistían en localizar en un texto o en una tabla información relevante para la pregunta. Cinco preguntas requerían integrar información que se encuentra bien en varios párrafos de un texto, o bien en varias columnas o filas de una tabla. Por último, dos preguntas requerían reflexionar sobre información que aparecía en un texto de la web. Para cada pregunta se distinguieron las páginas relevantes (aquellas que contenían información útil para responder la pregunta), de las irre- 
levantes (aquellas sin información pertinente para resolver la tarea).

La administración de las preguntas, el control del tiempo límite para responder a las mismas ( 3 minutos) y el registro tanto de las respuestas a las mismas como de las acciones de los participantes sobre el sitio Web, se realizó mediante una herramienta diseñada para tal fin en el lenguaje Visual Basic. El programa imitaba en apariencia a un navegador convencional (Internet Explorer), y permitía que los alumnos leye- ran la pregunta, consultaran la web y regresan a la pregunta, tantas veces como necesitasen o quisieran. Después de seleccionar la respuesta que consideran correcta, podían pasar a la siguiente pregunta y repetir el proceso.

Un análisis conjunto de las preguntas reflejó un alpha de Cronbach de .68, nivel aceptable de fiabilidad. El índice de dificultad promedio fue de $.60(D T=.18)$ (mínimo .50, máximo.92).

Tabla 1. Preguntas de lectura digital utilizadas en el estudio.

ID Item Descripción de la pregunta $\quad$ Pasos requeridos para resolver la pregunta

1 Localización informa- ¿En qué idioma encontrarás primero el videojuego Clic "Tecnología" > Clic "Videojuegos" > Clic "Próximos ción en un texto "El Profesor Layton y la Máscara Milagrosa de estrenos" > Scroll > Localizar información en el texto 2010? [Evitar imagen distractora]

2 Localizar información Si tengo entre 12 y 16 años ¿Cuánto puede costar- Clic "Cursos" > Clic "Danza" > Clic "Baile en la Fundaen tabla de datos me apuntarme a la Escuela de Baile del Complejo ción Deportiva Municipal" > Localizar información tabla Deportivo Cultural Petxina? (columna precio)

3 Integrar información ¿Por qué es importante controlar la respiración an- Clic "Salud" > Clic "Mis sentimientos" > Clic "Tienes un de un texto te el estrés de un examen? el texto

4 Localizar información Kobe Bryant es el jugador de baloncesto favorito Clic "Deportes" > Clic "Baloncesto" > Clic "La elección en el texto de tu novia. Has averiguado que fue seleccionado de jugadores en la NBA" > Localizar información en el para la NBA cuando... texto [Evitar video distractor]

5 Reflexión sobre la in- En el huerto experimental de tu centro estáis culti- Clic "Medioambiente" > Clic "Plantas protegidas" > Clic formación que apare- vando algunas plantas en peligro de extinción "Flora española en peligro de extinción" > Reflexionar soce en el texto ¿Cómo podríais cultivar el Trébol de Agua? bre información del texto

6 Integrar información ¿Cuál es el videojuego de Pokemon más vendido? Clic "Tecnología" > Clic "Videojuegos" > Clic "Próximos en tabla de datos estrenos" > Clic "Lista de ventas" > Integrar información tabla (columnas ventas y nombre)

7 Integrar información De todos los cursos de fotografía que aparecen en Clic "Cursos" > Clic "Artes plásticas" > Clic "Cursos de en tabla de datos la web ¿Cuál crees que ofrece un contenido con fotografía" > Scroll > Integrar información tabla (columna más prácticas reales? duración)

8 Localizar información Tu hermano tiene 12 años y es un excelente juga- Clic "Deportes" > Clic "Fútbol" (Deportes) $>$ Clic "La en un texto dor de fútbol. Le gustaría entrar en el centro de al- cantera del F.C. Barcelona" > Localizar información en el to rendimiento "La masía" ¿Cumplirá el requisito texto [Evitar video distractor] de edad?

9 Localizar información Los sms que ofrecen descargas de contenido mul- Clic "Tecnología" > Clic "Móviles" > Clic "Algunos conen un texto timedia suelen ser... $\quad$ sejos de seguridad" $>$ Localizar información en el texto [Evitar video distractor]

10 Integrar información Siempre que uses materiales reciclados para hacer Clic "Medioambiente" > Clic "Reciclaje" > Clic "Manualide un texto manualidades, se recomienda que: dades para reciclar" > Scroll > Integrar información entre párrafos

11 Reflexión sobre la in- Tu vecino Pedro hace escalada. Tiene material que Clic "Deportes" > Clic "Equipo y material deportivo" > formación que apare- ya no utiliza y que le gustaría vender a través de un Clic "Trocathlon vuelve a Decathlon" > Scroll > Reflexioce en el texto servicio que ofrecen en Decathlon. Ayúdale a ave- nar sobre información del texto riguar si le costará algo

12 Localizar información ¿Cuál puede ser la razón de que el ordenador inicie Clic "Tecnología" > Clic "Ordenadores" > Clic "Probleen un texto aplicaciones por sí solo?

mas más frecuentes" > Localizar información en el texto [Evitar video distractor]

Nota. Tras leer cada pregunta, los estudiantes debían clicar en un botón con la leyenda "Ir a las páginas web". Asimismo, para responder a las preguntas los estudiantes debían clicar en un botón con la leyenda "Responder a la pregunta" situado en la parte superior derecha del navegador.

\section{Test de habilidades digitales}

Se utilizó el Test de Habilidades Digitales desarrollado como parte del proyecto de investigación en el que se engloba el presente estudio. El test mide las habilidades operativas básicas de internet en cuanto al uso del navegador, uso de menús, navegación hipertextual y envío de e-mails. Es un test de ejecución por lo que los participantes deben realizar, en un entorno Web controlado, una serie de 14 tareas (ver tabla 2 para un análisis detallado de las tareas) tales como usar el botón de atrás del navegador, archivar páginas en favoritos, seccionar los enlaces activos de una página Web, seleccionar una sección en un menú dinámico, o enviar un email con copia. Los estudiantes disponían de 60 segundos 
para completar cada una de las tareas. En un pase piloto se evaluaron a 71 alumnos de $2^{\circ}$ y $3^{\circ}$ de ESO de un centro concertado de la ciudad de Valencia. Los participantes fueron distintos a la muestra del estudio principal. En el pase piloto se obtuvo un índice de fiabilidad aceptable (alpha de Cronbach $=.87)$, con un índice de dificultad promedio de .68 $(D T=.41)$ (mínimo .43, máximo .89).

Tabla 2. Ítems del test de habilidades digitales, y media de aciertos (sobre 1) y desviaciones típicas (en paréntesis) por grupo.

\begin{tabular}{|c|c|c|c|c|c|c|}
\hline ID & Item & $\begin{array}{l}\text { Entorno } \\
\text { simulado }\end{array}$ & Descripción de la tarea & Pasos requeridos para resolver la tarea & $\begin{array}{ll}\text { Aciertos } & 5 \\
\text { Primaria } & \end{array}$ & $\begin{array}{l}\text { Aciertos } 3 \\
\text { ESO }\end{array}$ \\
\hline 1 & Botón home & $\begin{array}{l}\text { Navegador } \\
\text { Web }\end{array}$ & $\begin{array}{l}\text { Vuelve a la página inicial del na- } \\
\text { vegador. }\end{array}$ & Clic en botón "Home" & $0.05(0.23)$ & $0.32(0.48)$ \\
\hline 2 & $\begin{array}{l}\text { Botón favo- } \\
\text { ritos }\end{array}$ & $\begin{array}{l}\text { Navegador } \\
\text { Web }\end{array}$ & $\begin{array}{l}\text { Guarda la dirección URL en el } \\
\text { navegador para visitarla poste- } \\
\text { riormente. }\end{array}$ & Clic en botón "Añadir a favoritos" & $0.11(0.32)$ & $0.61(0.5)$ \\
\hline 3 & $\begin{array}{l}\text { Botón nueva } \\
\text { pestaña }\end{array}$ & $\begin{array}{l}\text { Navegador } \\
\text { Web }\end{array}$ & $\begin{array}{l}\text { Abre una nueva pestaña del na- } \\
\text { vegador. }\end{array}$ & Clic en botón "Nueva pestaña" & $0.28(0.46)$ & $0.81(0.4)$ \\
\hline 4 & $\begin{array}{l}\text { Tabla de } \\
\text { contenidos }\end{array}$ & $\begin{array}{l}\text { Enciclope- } \\
\text { dia digital }\end{array}$ & $\begin{array}{l}\text { Ve directamente a la informa- } \\
\text { ción sobre "mamíferos". }\end{array}$ & Clic en hiperenlace "Indice de contenidos" & $0.44(0.51)$ & $0.55(0.51)$ \\
\hline 5 & $\begin{array}{l}\text { Hiperenlace } \\
\text { sin distracto- } \\
\text { res }\end{array}$ & Página web & $\begin{array}{l}\text { Ve al final del texto para ver el } \\
\text { nombre de la autora. }\end{array}$ & Clic en hiperenlace "Seguir leyendo" & $0.72(0.46)$ & $0.94(0.25)$ \\
\hline 6 & $\begin{array}{l}\text { Hiperenlace } \\
\text { con distrac- } \\
\text { tores }\end{array}$ & Página web & $\begin{array}{l}\text { Entra en la sección de la página } \\
\text { para enviar tu propia foto. }\end{array}$ & $\begin{array}{l}\text { Clic en hiperenlace "Envía tu propia foto- } \\
\text { grafía" }\end{array}$ & $0.83(0.38)$ & $1(0)$ \\
\hline 7 & Scroll & Página web & $\begin{array}{l}\text { Busca la información de contac- } \\
\text { to de la página. }\end{array}$ & $\begin{array}{l}\text { Scroll hasta el final de la página. Clic en } \\
\text { hiperenlace "Contactar" }\end{array}$ & $0.56(0.51)$ & $0.97(0.18)$ \\
\hline 8 & $\begin{array}{l}\text { Valoración } \\
\text { social }\end{array}$ & $\begin{array}{l}\text { Librería en } \\
\text { línea }\end{array}$ & $\begin{array}{l}\text { Haz click sobre el libro mejor } \\
\text { valorado por los lectores. }\end{array}$ & $\begin{array}{l}\text { Clic sobre item con mayor número de "es- } \\
\text { trellas" }\end{array}$ & $0.5(0.51)$ & $0.74(0.44)$ \\
\hline 9 & $\begin{array}{l}\text { Entrar co- } \\
\text { rreo }\end{array}$ & Correo web & $\begin{array}{l}\text { Entra en tu correo con la cuenta } \\
\text { "invitado@correo.es" y la con- } \\
\text { traseña "1234". }\end{array}$ & $\begin{array}{l}\text { Introducir información correspondiente } \\
\text { en los campos "usuario" y "contraseña" > } \\
\text { Clic en botón "entrar" }\end{array}$ & $0.33(0.49)$ & $0.71(0.46)$ \\
\hline 10 & $\begin{array}{l}\text { Enviar co- } \\
\text { rreo simple }\end{array}$ & Correo web & $\begin{array}{l}\text { Envía este mensaje tal y como } \\
\text { está a la dirección "ami- } \\
\text { go@correo.uv.es" }\end{array}$ & $\begin{array}{l}\text { Introducir dirección en apartado "Para" > } \\
\text { Clic en botón "Enviar". }\end{array}$ & $0.28(0.46)$ & $0.48(0.51)$ \\
\hline 11 & $\begin{array}{l}\text { Enviar co- } \\
\text { rreo comple- } \\
\text { jo }\end{array}$ & Correo web & $\begin{array}{l}\text { Envía este mensaje tal y como } \\
\text { está a la dirección "profe- } \\
\text { sor@correo.uv.es" y envía una } \\
\text { copia a "alumno@correo.uv.es" }\end{array}$ & $\begin{array}{l}\text { Introducir direcciones en apartados "Para" } \\
\text { y "CC" > Clic en botón "Enviar". }\end{array}$ & $0.28(0.46)$ & $0.26(0.44)$ \\
\hline 12 & Abrir correo & Correo web & $\begin{array}{l}\text { Acaba de llegarte un mensaje. } \\
\text { Abrelo. }\end{array}$ & Clic en botón "Band. entrada (1)" & $0.67(0.49)$ & $0.97(0.18)$ \\
\hline 13 & $\begin{array}{l}\text { Uso del ra- } \\
\text { tón }\end{array}$ & $\begin{array}{l}\text { Menú di- } \\
\text { námico }\end{array}$ & $\begin{array}{l}\text { Ve a la opción "B1" a través de } \\
\text { la ruta "Menú" - "Opción B" - } \\
\text { "Página B1" }\end{array}$ & $\begin{array}{l}\text { Colocar el puntero del ratón sobre la op- } \\
\text { ción "Menú" > "Opción B" > "Página } \\
\text { B1" [El menú dinámico solo se despliega } \\
\text { si el puntero del ratón permanece sobre la } \\
\text { opción correspondiente. Este menú no } \\
\text { reacciona ante los clics de ratón.] }\end{array}$ & $0.56(0.51)$ & $0.9(0.3)$ \\
\hline 14 & $\begin{array}{l}\text { Introducir } \\
\text { datos }\end{array}$ & $\begin{array}{l}\text { Converti- } \\
\text { dor }\end{array}$ & $\begin{array}{l}\text { Quieres comprar un juego de } \\
\text { consola por internet cuyo precio } \\
\text { es de } 45 \text { dólares USA. Utiliza el } \\
\text { siguiente convertidor de mone- } \\
\text { da para saber cuántos euros son } \\
45 \text { dólares USA. }\end{array}$ & $\begin{array}{l}\text { Introducir información en apartado "Dó- } \\
\text { lares USA" > Clic en botón "Convertir". }\end{array}$ & $0.67(0.49)$ & $0.97(0.18)$ \\
\hline
\end{tabular}

\section{Test de comprensión lectora}

Para evaluar la comprensión lectora se utilizó el Test de Procesos de Comprensión (TPC) de Vidal-Abarca y col. (2007). El TPC se dirige a niños entre 10 y 16 años. Su aplicación es colectiva en formato tradicional (lápiz y papel). El TPC contiene 2 textos expositivos acompañados de 10 preguntas cada uno. Las preguntas evalúan procesos de comprensión relevantes según la teoría de Kintsch (1998): la cap- tación de ideas explícitas, la capacidad de realizar inferencias anafóricas y basadas en el conocimiento previo, y la elaboración de macroideas. El test ha sido baremado para distintos niveles educativos (desde $5^{\circ}$ Primaria hasta $4^{\circ}$ de ESO).

\section{Procedimiento}

El estudio tuvo lugar en dos sesiones de aproximadamente 50 minutos cada una. En una primera sesión los 
alumnos completaron el test de comprensión lectora en respectivas aulas y en horario escolar. En una segunda sesión, que tuvo lugar dos semanas después de la primera, los alumnos completaron el test de habilidades digitales y las tareas de lectura digital en las salas de ordenadores del propio centro.

A los alumnos se les informó de que iban a participar en un estudio de la Universidad sobre cómo manejaban la información que aparecía en las páginas Web, contestando a las preguntas que se les presentaría a través del ordenador. Se les animó a contestar lo que considerasen correcto. Para cada una de las dos tareas se incluyó una página de inicio introductoria donde se volvían a mostrar las instrucciones. Para la tarea experimental con el portal web las preguntas se presentaron en orden aleatorio por participante a fin de evitar un efecto de orden y que los alumnos copiaran las respuestas. No se permitió al alumno pasar a la pregunta siguiente sin antes haber contestado la actual.

\section{Resultados}

\section{Análisis descriptivos}

En primer lugar se analizaron los niveles de ejecución de los estudiantes en las distintas tareas en función del curso $\left(5^{\circ}\right.$ Primaria o $3^{\circ}$ de ESO), mediante pruebas t de comparación de medias (Tabla 3). Cuando las varianzas fueron desiguales se corrigieron los grados de libertad correspondientes. Como era de esperar, los estudiantes de $3^{\circ}$ de ESO obtuvieron mayores puntuaciones que los de $5^{\circ}$ de Primaria en habilidades digitales, comprensión lectora y lectura digital. Concretamente, en cuanto a las tareas de lectura digital, los estudiantes de $3^{\circ}$ de ESO fueron mejores en los tres tipos de preguntas: localización, integración y reflexión. En cuanto a las tareas de habilidades digitales, los alumnos de $3^{\circ}$ de ESO obtuvieron una mejor puntuación en todas las tareas (todas las $t_{\mathrm{s}}>2.02$ ), excepto en los ítems 4 (tabla de contenidos), 8 (valoración social), 10 y 11 (enviar correo simple / complejo) (todas las ts $<1.59$ ) (ver medias y desviaciones típicas en la Tabla 2).

Asimismo, los estudiantes de $3^{\circ}$ de ESO mostraron una mejor navegación, como se desprende de un mayor promedio de visitas a páginas relevantes, un mayor tiempo promedio de visitas a páginas relevantes y a un menor número de irrelevantes.

A nivel cualitativo, en cuanto a la comprensión lectora evaluada por el TPC (Vidal-Abarca y cols., 2007), los resultados indicaron que las puntuaciones directas equivalían a percentiles normales o incluso altos de ejecución (la media situaba a los grupos en un percentil 60). En cuanto a las habilidades digitales, el nivel de ejecución fue entre medio y elevado, pero en ninguno de los cursos se obtuvo un efecto techo. En la misma línea, los datos de las tareas de lectura digital (porcentaje de aciertos) fueron intermedios. Nótese que en las dos últimas tareas la interpretación sobre el nivel de ejecución es especulativa, puesto que no se trata de prue- bas estandarizadas. Por último, los datos de navegación indicaron que los alumnos de $3^{\circ}$ de ESO visitaban y estaban más tiempo en promedio en un mayor número de páginas relevantes que irrelevantes, un patrón que se revertía para los estudiantes de $5^{\circ}$ de Primaria. Los estudiantes más jóvenes tuvieron dificultad en mantener un patrón de navegación en páginas relevantes, y con mayor frecuencia navegaron durante gran tiempo por páginas con poca probabilidad de incluir información útil para su tarea.

Tabla 3. Niveles de ejecución media por curso en las distintas tareas realizadas, así como en las variables de navegación de las tareas de lectura digital (en paréntesis se muestran las desviaciones típicas).

\begin{tabular}{lccc}
\hline Medida & $5^{\circ}$ Primaria & $3^{\circ}$ ESO & \\
\hline Habilidades digitales & $.5(.23)$ & $.79(.14)$ & $t(52)=-4.93^{* *}$ \\
Comprensión lectora & $.52(.12)$ & $.74(.19)$ & $t(52)=-4.98^{* *}$ \\
Lectura digital (LD) & $.45(.15)$ & $.70(.17)$ & $t(52)=-4.98^{* *}$ \\
$\quad$ LD - localización & $.47(.20)$ & $.72(.10)$ & $t(23.31)=4.77^{* *}$ \\
$\quad$ LD- integración & $.46(.18)$ & $.69(.24)$ & $t(52)=3.45^{* *}$ \\
$\quad$ LD- reflexión & $.44(.29)$ & $.62(.28)$ & $t(52)=1.99^{*}$ \\
Visitas promedio páginas & $1.19(1.11)$ & 2.41 & $t(52)=-4.24^{* *}$ \\
relevantes & & $(0.71)$ & \\
Visitas promedio páginas & $2.98(1.72)$ & $2.0(1.51)$ & $t(52)=-1.97 *$ \\
irrelevantes & & & \\
Tiempo promedio páginas & $6.28(5.45)$ & 21.06 & $t(52)=-6.50 * *$ \\
relevantes (sec) & & $(8.91)$ & \\
Tiempo promedio páginas $16.46(20.64)$ & 15.26 & $t<1$ \\
irrelevantes (sec) & & $(7.31)$ & \\
\hline Nota: ${ }^{*} p .05, * * p<.01$. & & &
\end{tabular}

\section{Efectos de comprensión lectora, habilidades digita- les y navegación}

En relación a nuestro primer objetivo, la replicación de los efectos positivos de la comprensión lectora, habilidades digitales y navegación en las tareas de navegación, se realizaron una serie de correlaciones Pearson entre las habilidades medidas y las variables de navegación (Tabla 4). Las puntuaciones en la prueba de lectura digital correlacionaron positivamente con las puntuaciones en los test de habilidades digitales y de comprensión, así como con las visitas y tiempos promedio a páginas relevantes. Por su parte, las habilidades digitales correlacionaron positivamente con la comprensión lectora, así como con las variables de navegación por páginas relevantes.

Este patrón de resultados replica en gran medida los efectos positivos en tareas de lectura digital de las habilidades digitales (Goldhammer y cols., 2013; Hahnel y cols., 2016; Van Deursen y Van Dijk, 2009), comprensión lectora y navegación (Coiro, 2011; OECD, 2011; Salmerón y García, 2011). Asimismo, los resultados clarifican que las habilidades digitales están relacionadas con la utilización de una estrategia de navegación óptima por páginas relevantes para contestar la tarea. Sin embargo, la correlación entre las habilidades digitales y la comprensión lectora no permiten descartar la hipótesis de que en realidad el efecto encontrado en la ejecución en las tareas de lectura digital se deba únicamente a una de ellas. Para descartar esta hipótesis llevamos a cabo un 
análisis de regresión en el que se predijo la ejecución en las tareas de lectura digital a partir de las variables de habilidades y navegación.

Tabla 4. Correlaciones Pearson entre en las distintas tareas realizadas, así como en las variables de navegación de las tareas de lectura digital.

\begin{tabular}{|c|c|c|c|c|c|}
\hline Medida & 2 & 3 & 4 & 5 & 6 \\
\hline 1. Habilidades digitales & - & & & & \\
\hline 2. Comprensión lectora & $.47 * *$ & & & & \\
\hline 3. Lectura digital & $.57 * * .54 * *$ & - & & & \\
\hline $\begin{array}{l}\text { 4. Visitas promedio páginas } \\
\text { relevantes }\end{array}$ & $.47 * * .44 * *$ & $.58 *$ & $*_{\text {* }}$ & & \\
\hline $\begin{array}{l}\text { 5. Visitas promedio páginas } \\
\text { irrelevantes }\end{array}$ & -.10 & -.33 & $k^{*}-.02$ & - & \\
\hline $\begin{array}{l}\text { 6. Tiempo promedio pág. } \\
\text { relevantes (sec) }\end{array}$ & $.48 * * .58 * *$ & $.54 *$ & $* .66 * *$ & $*-.41 * *$ & - \\
\hline $\begin{array}{l}\text { 7. Tiempo promedio pág. } \\
\text { irrelevantes (sec) }\end{array}$ & $-.08 \quad .12$ & -.17 & -.24 & $.32 *$ & -.12 \\
\hline
\end{tabular}

\section{Relación entre habilidades digitales y lectura digital}

Como se recordará nuestro segundo objetivo consistía en analizar la relación existente entre las habilidades digitales en su impacto en las tareas de lectura digital, controlando el posible efecto de las habilidades de comprensión en papel y de la navegación. Con tal fin se llevó a cabo un análisis de regresión múltiple con las puntuaciones en preguntas de lectura digital como variable dependiente. Como predictores se incluyeron el curso, los valores de los test de habilidades digitales y de comprensión lectora, y el número promedio de páginas relevantes e irrelevantes visitadas. Dada la gran relación entre las medidas de tiempo y visitas se optó por incluir únicamente las últimas en el modelo de regresión. Para facilitar la interpretación de los resultados todas las puntuaciones de los alumnos se transformaron en puntuaciones $z$. La variable curso ( $5^{\mathrm{a}}$ primaria y $\left.3^{\mathrm{a}} \mathrm{ESO}\right)$ se introdujo como variable dummy.

El modelo de regresión resultó ser significativo, explicando el $55 \%$ de la varianza del éxito en las preguntas de lectura digital (Tabla 5). Concretamente, los dos predictores de habilidades (digitales y de comprensión) contribuyeron positiva y significativamente al modelo. Los valores de las betas indicaban que la ejecución en tareas de lectura digital se incrementaban en un .26 o .29 desviación estándar por cada aumento de una desviación estándar en los test de habilidades digitales y de comprensión lectora en papel, respetivamente. Asimismo, los dos predictores de navegación (promedio de visitas a páginas relevantes e irrelevantes), contribuyeron significativamente al modelo. La ejecución en las tareas de lectura digital aumentó un .33 o disminuyó un .32 por cada aumento de una desviación estándar en el número promedio de visitas a páginas relevantes e irrelevantes, respectivamente. Aunque la variable curso fue marginalmente significativa si sólo se consideraban los predictores de habilidades, $t(45)=1.70, p=.08$, su efecto se desvaneció al intro- ducir en el modelo de regresión los predictores de navegación (ver Tabla 5).

Tabla 5. Resumen del análisis de regresión múltiple para los efectos de curso, habilidades digitales y de comprensión lectora, y navegación en tareas de lectura digital.

\begin{tabular}{|c|c|c|c|}
\hline & $B$ & SEB & $T$ \\
\hline Intercepto (B0) & .01 & .10 & \\
\hline Curso $^{\text {a }}$ & -.03 & .31 & -.09 \\
\hline Habilidades digitales $b$ & .26 & .13 & $2.02 *$ \\
\hline Habilidades de comprensión ${ }^{b}$ & .29 & .12 & $2.43 *$ \\
\hline Visitas promedio páginas relevantes ${ }^{b}$ & .33 & .12 & $2.73 * *$ \\
\hline Visitas promedio páginas irrelevantes $\mathrm{b}$ & -.32 & .10 & $-3.02 * *$ \\
\hline Encaje del modelo & \multicolumn{3}{|c|}{$\mathrm{R}^{2}$ corregida $=.55$} \\
\hline Test omnibus & \multicolumn{3}{|c|}{$F(5,43)=12.84, p<.01$} \\
\hline
\end{tabular}

Para ahondar en la naturaleza de las relaciones entre las distintos tipos de tareas de lectura digital (localización, integración y reflexión) y el resto de variables individuales, se realizaron una serie de correlaciones parciales. En primer lugar, se exploró la correlación entre las habilidades de comprensión y los tres tipos de tareas de lectura digital, controlando la puntuación en el test de habilidades digitales. Los resultados mostraron una relación significativa entre las habilidades de comprensión y las preguntas de integración digital $(r(52)=.32, p<.05)$, pero no para las preguntas de localización $(r(52)=.04, p=.79)$, o reflexión $(r(52)=-.13, p=.36)$. En segundo lugar, se analizó la correlación entre las habilidades básicas de navegación y los tres tipos de tareas de lectura digital, esta vez controlando la puntuación en el test de habilidades de comprensión. Las correlaciones mostraron resultados significativos para los tres tipos de pregunta utilizados: localización $(r(52)=.46, \quad p<.01)$, integración $(r(52)=.74, p<.01)$, y reflexión $(r(52)=-.57, p<.01)$. Por último, se trató de esclarecer la relación entre las habilidades digitales básicas y el éxito la lectura digital. Para ello se computaron una serie de correlaciones parciales entre los distintos ítems de la prueba de habilidades digitales básicas y la puntuación global en la prueba de lectura digital, controlando las habilidades de comprensión de los estudiantes. 5 de los 14 ítems correlacionaron significativamente (todas las rs > .29): uso del navegador web (ítems 2 y 3), selección de hiperenlaces con distractores (ítem 6), envío de correo simple (ítem 10) y uso del ratón en un menú dinámico (ítem 13).

En resumen, para estos grupos de estudiantes de educación primaria y secundaria, las habilidades digitales y de comprensión lectora, así como la navegación, predecían de manera independiente y sustancial el éxito en las tareas de lectura digital, independientemente del curso en el que se encuentren. Como muestra de su relevancia, la magnitud de la relación entre las habilidades digitales (como el uso del navegador, menús, ...) y la ejecución de las tareas de lectura digital es comparable a la relación de ésta última con las habilidades de comprensión. 


\section{Conclusiones}

Los resultados constatan el efecto positivo de las habilidades digitales básicas en la lectura digital en tareas tipo PISA en una población de estudiantes de primaria y secundaria. Este patrón de resultados replica el efecto reportado previamente en adultos (van Deursen y van Dijk, 2009), y delimita los resultados encontrados con estudiantes de secundaria (Goldhammer y cols., 2013; Hahnel y cols., 2016): el efecto de las habilidades digitales básicas en las tareas de lectura digital, particularmente aquellas relacionadas con el manejo del navegador, uso de menús complejos y de hiperenlaces en webs complejas, es independiente tanto del impacto de la comprensión lectora de los estudiantes como del efecto del patrón de navegación seguido por los mismos. De hecho, en nuestro estudio el tamaño del efecto de las habilidades digitales básicas en la ejecución de tareas de lectura digital es similar al producido por la comprensión lectora, uno de los factores más relevantes en la lectura digital encontrados hasta la fecha (Coiro, 2011; OECD, 2011; Salmerón y García, 2011). Asimismo, los resultados sugieren que las habilidades digitales básicas facilitan la lectura digital en sus diferentes vertientes, ya sea cuando el objetivo es localizar, integrar o reflexionar sobre información en la Web.

La relación positiva entre las habilidades digitales y la lectura digital se ha encontrado tanto en la muestra de estudiantes de $5^{\circ}$ de Primaria como en la de $3^{\circ}$ de Secundaria. De hecho, las puntuaciones en las tareas de ambas habilidades distan de ser perfectas en los estudiantes mayores, lo que sugiere que la relación entre ambas podría mantenerse durante los estudios de bachillerato y en la universidad (cf. van Deursen y Van Dijk, 2009, 2009). Asimismo, la constatación de que las habilidades digitales básicas influyen en la lectura digital de forma independiente de la comprensión lectora señala la necesidad de instruir ambas competencias para favorecer el desarrollo de la competencia en lectura digital.

Por último, la correlación positiva entre las habilidades digitales y el número de visitas y tiempo promedio a páginas relevantes sugieren que aquellas podrían favorecer la navegación selectiva por páginas relevantes para la tarea, lo que de por sí tiene un efecto positivo en la lectura digital tanto en estudiantes de educación secundaria (OECD, 2011) como en universitarios (Naumann, Richter, Flender, Christmann, \& Groeben, 2007; Naumann, Richter, Christmann, \& Groeben, 2008). Nuestros resultados replican y extienden este efecto en una muestra de estudiantes más jóvenes $\left(5^{\circ}\right.$ de Primaria).

\section{Implicaciones para la evaluación y la instrucción de la competencia en lectura digital}

La utilización de un test objetivo para evaluar las habilidades digitales de los estudiantes ha permitido clarificar el vínculo entre este factor y la competencia en lectura digital. Como se apuntó en la introducción, la evaluación de las habilidades digitales mediante índices como la frecuencia de uso de ordenadores puede dar lugar a patrones poco verosímiles y con una difícil interpretación (OECD, 2011). La evaluación objetiva de las habilidades digitales básicas se podría incluir en evaluaciones a gran escala de la competencia digital como PISA, y de esa forma se podría delimitar qué parte de la ejecución en dicha competencia se debe en realidad a una falta de habilidades digitales básicas. Por ejemplo, alumnos de países con un bajo acceso a ordenadores podrían obtener puntuaciones bajas en PISA no tanto por su falta de habilidades para leer estratégicamente en Internet, sino simplemente por su falta de habilidades digitales para implementar dichas estrategias en el medio electrónico.

Los resultados de nuestro estudio también tienen implicaciones sobre la instrucción de las competencias digitales. El currículo oficial de educación primaria de España establece como necesario trabajar con la comprensión lectora y con las tecnologías de la información y la educación, en todas las áreas (BOE n. 173 del 20 de julio de 2007. Orden ECI/2211/2007 del 12 de julio). Además, establece como uno de los objetivos principales de esta etapa "Iniciarse en la utilización, para el aprendizaje, de las tecnologías de la información y la comunicación desarrollando un espíritu crítico ante los mensajes que reciben y elaboran". El currículo oficial de primaria establece que un alumno, al terminar esta etapa debe haber desarrollado una serie de competencias, entre ellas, el tratamiento de la información y competencia digital que consiste en... "disponer de habilidades para buscar, obtener, procesar y comunicar información y para transformarla en conocimiento...". La situación es semejante en la Educación Secundaria Obligatoria. En el currículo oficial de esta etapa (BOE núm. 35 del 10 de febrero de 2004, Real Decreto 116/2004 de 23 de enero) se plantean como objetivos, entre otros, desarrollar destrezas básicas en la utilización de las fuentes de información para adquirir, con sentido crítico, nuevos conocimientos y adquirir una preparación básica en el campo de las tecnologías, fundamentalmente mediante la adquisición de las destrezas relacionadas con las tecnologías de la información y de la comunicación, a fin de usarlas en el proceso de aprendizaje para encontrar, analizar, intercambiar y presentar la información y el conocimiento adquiridos. Según nuestro estudio, creemos que el profesorado, o los colegios al diseñar su carácter propio, deberían incluir en su programación la enseñanza específica del conocimiento y uso de las herramientas digitales que influyen en la comprensión digital.

Además, de nuestro estudio parece desprenderse que la competencia digital en cuanto a localizar, integrar y reflexionar sobre la información que aparece en el hipertexto, así como la competencia en las habilidades digitales necesarias, como usar los hiperenlaces, el navegador..., debería fomentarse más explícitamente en nuestros currículos oficiales, para reducir el riesgo de que quedara en manos del propio profesorado qué parte del tiempo dedicar a la construcción del conocimiento a través de estos medios. Como se apuntó en la introducción, existe la creencia generalizada de que los estudiantes de hoy en día pertenecen a una generación de 'na- 
tivos digitales' (Prensky, 2001), por lo que se tiende a asumir que estos alumnos ya poseen las habilidades digitales básicas para manejarse en las tareas de lectura digital como las planteadas por PISA. Nuestros resultados chocan frontalmente con esta visión del alumnado, y enfatizan la necesidad de abordar directamente la instrucción de estas habilidades para evitar una mayor brecha digital entre los estudiantes con altas y bajas habilidades. Como sugieren Brand-Gruwel y cols. (2009), las habilidades digitales básicas son un prerrequisito para poder adquirir las competencias de lectura digital.

Igualmente, un test de evaluación de las habilidades digitales como el aplicado en este estudio podría utilizarse para adaptar la instrucción en entornos digitales (e.g. uso de los WebQuest en el aula). Los estudiantes que ya de por sí tropiezan con operaciones básicas como el uso del navegador podrían disponer de más tiempo para la realización de la tarea.

\section{Referencias}

Bennett, S., y Maton, K. (2010). Beyond the 'digital natives' debate: Towards a more nuanced understanding of students' technology experiences. Journal of Computer Assisted Learning, 26(5), 321-331. doi: 10.1111/j.13652729.2010.00360.x

Bennett, S., Maton, K., y Kervin, L. (2008). The 'digital natives' debate: A critical review of the evidence. British Journal of Educational Technology, 39, 775-786. oi:10.1111/j.1467-8535.2007.00793.x

Brand-Gruwel, S., Wopereis, I., y Walraven, A. (2009). A descriptive model of information problem solving while using internet. Computers \& Education, 53(4), 1207-1217. doi: 10.1016/j.compedu.2009.06.004

Coiro, J., y Dobler, E. (2007). Exploring the online reading comprehension strategies used by sixth-grade skilled readers to search for and locate information on the Internet. Reading Research Quarterly, 42, 214-257. doi:10.1598/RRQ.42.2.2

Coiro, J. (2011). Predicting reading comprehension on the Internet: Contributions of offline reading skills, online reading skills, and prior knowledge. Journal of Literacy Research, 43(4), 352-392. doi:10.1177/1086296X11421979

Goldhammer, F., Naumann, J. y Keßel, Y. (2013). Assessing individual differences in basic computer skills. European Journal of Psychological Assessment, 29(4), 263-275. doi: 10.1027/1015-5759/a000153

Hahnel, C., Goldhammer, F., Johannes Naumann, J., \& Kröhne, U. (2016). Effects of linear reading, basic computer skills, evaluating online information, and navigation on reading digital text. Computers in Human Behavior, 55, 486-500. doi: 10.1016/j.chb.2015.09.042

Kintsch, W. (1998). Comprehension: A paradigm for cognition. New York: Cambridge university press.

Muriel, S. (2009). Impulsando la educación en red: retos y realidades. XXII Semana Monográfica de la Educación. Fundación Santillana. Recuperado de: http://www.fundacionsantillana.com/upload/ficheros/paginas/20090 6/xxii_semana monografica.pdf

Naumann, J., Richter, T., Christmann, U., y Groeben, N. (2008). Working memory capacity and reading skill moderate the effectiveness of strategy trainings in learning from hypertext. Learning and Individual Differences, 18, 197-213. doi:10.1016/j.lindif.2007.08.007

\section{Limitaciones e investigación futura}

El estudio tiene las limitaciones propias de un diseño experimental correlacional, lo que impide establecer vinculaciones causales entre las variables estudiadas. No obstante, la relación entre habilidades digitales y lectura digital va en la línea de estudios anteriores sobre la materia, por lo que la replicación de este efecto sugiere la existencia de un vínculo no trivial. En el futuro, estudios experimentales deberían constatar la existencia de causalidad, por ejemplo, observando el efecto de la instrucción en habilidades digitales básicas en un grupo de estudiantes en el desarrollo de las competencias de lectura digital, así como en su transferencia a la lectura digital fuera del aula (Salmerón, 2013).

Por último, el presente estudio utiliza una muestra reducida de estudiantes. Creemos que los resultados sobre la relación entre las habilidades digitales y la lectura digital encontrados en este estudio podrían servir de aval para la inclusión de una medida objetiva de habilidades digitales en evaluaciones a gran escala de la competencia digital en el futuro.

Agradecimientos.- Esta investigación ha sido financiada por la Secretaría General de Universidades (proyecto EDU2011-25885).

Naumann, J., Richter, T., Flender, J., Christmann, U., \& Groeben, N. (2007). Signaling in expository hypertexts compensates for deficits in reading skill. Journal of Educational Psychology, 99(4), 791-807. doi: 10.1037/00220663.99.4.791

OECD (2009). PISA 2009 Assessment framework - Key competencies in reading, mathematics, and science. Paris, France: OECD. Retrieved from: http://www.oecd.org/pisa/pisaproducts/44455820.pdf

OECD (2011). PISA 2009 Results: Students on Line: Digital Technologies and Performance (Volume VI). Paris, France: OECD. Retrieved from: http://browse.oecdbookshop.org/oecd/pdfs/free/9811031e.pdf

Prensky, M. (2001). Digital natives, digital immigrants part 1. On the Horizon, 9, 1 - 6. doi : 10.1108/10748120110424816

Richter, T., Naumann, J., \& Horz, H. (2010). Eine revidierte Fassung des Inventars zur Computerbildung (INCOBI-R). Zeitschrift für pädagogische Psychologie, 24(1), 23-37. 10.1024/1010-0652/a000002

Salmerón, L. (2013). Actividades que promueven la transferencia de los aprendizajes: una revisión de la literatura. Revista de Educación, volumen extraordinario 2013, 34-53. doi: 10.4438/1988-592X-RE-2013-EXT-253

Salmerón, L., y García V. (2001). Reading skills and children's navigation strategies in hypertext. Computers in Human Behavior, 27, 1143-1151. doi:10.1016/j.chb.2010.12.008

Segura, M (2009). Las TIC en la educación: panorama internacional y situación española. XXII Semana Monográfica de la Educación. Fundación Santillana. Recuperado

de: http://www.fundacionsantillana.com/upload/ficheros/paginas/20090 6/xxii semana monografica.pdf

Tu, Y. W., Shih, M., y Tsai, C. C. (2008). Eighth graders' web searching strategies and outcomes: The role of task types, web experiences and epistemological beliefs. Computers \& Education, 51(3), 1142-1153. doi:10.1016/j.compedu.2007.11.003

Van Deursen, A. J., y Van Dijk, J. A. (2009). Using the Internet: Skill related problems in users' online behavior. Interacting with Computers, 21(5), 393402. doi: 10.1016/j.intcom.2009.06.005

Vidal-Abarca, E., Gilabert, R. Martínez, T., Sellés. P., Abad. N., y Ferrer, C. (2007). TEC. Test de Estrategias de Comprensión. Madrid, ICCE. 